\title{
An Integral Boundary Value Problem of Fractional Differential Equations with a Sign-Changed Parameter in Banach Spaces
}

\author{
Chen Yang $\mathbb{D},{ }^{1}$ Yaru Guo, ${ }^{2}$ and Chengbo Zhai $\mathbb{D}^{2}$ \\ ${ }^{1}$ Basic Course Department, Business College of Shanxi University, Taiyuan, Shanxi 030031, China \\ ${ }^{2}$ School of Mathematical Sciences, Shanxi University, Taiyuan 030006, Shanxi, China \\ Correspondence should be addressed to Chen Yang; yangchen0809@126.com
}

Received 23 July 2020; Revised 21 January 2021; Accepted 28 January 2021; Published 11 February 2021

Academic Editor: Atila Bueno

Copyright (c) 2021 Chen Yang et al. This is an open access article distributed under the Creative Commons Attribution License, which permits unrestricted use, distribution, and reproduction in any medium, provided the original work is properly cited.

This paper is to investigate the existence and uniqueness of solutions for an integral boundary value problem of new fractional differential equations with a sign-changed parameter in Banach spaces. The main used approach is a recent fixed point theorem of increasing $\Psi-(h, r)$-concave operators defined on ordered sets. In addition, we can present a monotone iterative scheme to approximate the unique solution. In the end, two simple examples are given to illustrate our main results.

\section{Introduction}

With the intensive development of theory and applications of fractional calculus, fractional differential equations have been paid great interest in many fields and thus different boundary conditions of fractional differential equations have also attracted much attention, see [1-42]. However, we can see that there are very few results reported on solutions for boundary value problems of fractional differential equations in Banach spaces.

In 2010, by using contraction mapping principle, fixed point index theory of completely continuous operator, and so on, Bai [2] obtained the existence results of positive solutions for the following three-point problem involving with fractional derivative:

$$
\begin{cases}D_{0^{+}}^{\beta} u(t)+f(t, u(t))=0, & 0<t<1, \\ u(0)=0, & \gamma u(\eta)=u(1),\end{cases}
$$

where $1<\beta \leq 2,0<\eta<1,0<\gamma \eta^{\beta-1}<1, D_{0^{+}}^{\beta}$ is the Riemann-Liouville (R-L for short) fractional derivative, and the function $f(t, u)$ is continuous on $[0,1] \times t[0,+\infty)$.

In 2014, by using Guo-Krasnoselskii fixed point theorem, Cabada and Hamdi [4] obtained the existence of positive solutions for the following fractional differential equations with an integral condition:

$$
\begin{cases}D_{0^{+}}^{\beta} u(t)+f(t, u(t))=0, & 0<t<1, \\ u(0)=u^{\prime}(0)=0, & u(1)=\rho \int_{0}^{1} u(t) \mathrm{d} t,\end{cases}
$$

where $2<\beta \leq 3,0<\rho<\beta, D_{0^{+}}^{\beta}$ is the $\mathbf{R}$ - $\mathbf{L}$ fractional derivative, and $f(t, u)$ is a continuous function.

As we know, most of the existing results of solutions for fractional differential equation boundary value problems have been obtained in real space $\mathbf{R}$. There exists very few papers studied in abstract spaces, and we can find $[3,9]$ and others.

In 2018, Chen and Gao [3] discussed the following problem of fractional differential equations in a Banach space $E$ :

$$
\begin{cases}-D_{0^{+}}^{\beta} u(t)=f(t, u(t)), & t \in J, \\ u(0)=u^{\prime}(0)=\theta, & u(1)=\rho \int_{0}^{1} u(t) \mathrm{d} t,\end{cases}
$$

where $2<\beta \leq 3,0<\rho<\beta, J=[0,1], D_{0^{+}}^{\beta}$ is the $\mathbf{R}$-L fractional derivative, $f: J \times K \longrightarrow K$ is continuous, here $K$ is a normal cone in $E$, and $\theta$ denotes the zero element of $E$. They 
obtained the existence results of positive solutions by using fixed point index theory of condensing mapping.

Motivated by these works, we consider the following new form of fractional differential equation with an integral boundary condition:

$$
\begin{cases}-D_{0^{+}}^{\beta} u(t)=f(t, u(t))+\gamma g(t), & t \in J, \\ u(0)=u^{\prime}(0)=\theta, & u(1)=\rho \int_{0}^{1} u(t) \mathrm{d} t\end{cases}
$$

where $2<\beta \leq 3,0<\rho<\beta$, and $\beta$ and $\rho$ are real numbers, $\gamma \in \mathbf{R}$ is a sign-changed parameter, $J=[0,1], D_{0^{+}}^{\beta}$ is the $\mathbf{R}-\mathbf{L}$ fractional derivative, $f: J \times K \longrightarrow K$ is continuous, $g: J \longrightarrow K$ is continuous, here $K$ is a normal cone in $E$, and $\theta$ is the zero element of $E$. From the literature, we know that problem (4) is a new form of fractional differential equations. Different from the previous works, we use a recent fixed point theorem for $\Psi-(h, r)$-concave operators defined in ordered set $P_{h, r}$ (see [43]) to study (4), and we obtain the existence and uniqueness of solution, while the uniqueness is not treated in the literature [3]. It should be pointed out that this method is interesting for solving nonlinear differential boundary value problems. In particular, we do not need the existence of upper-lower solutions, which is a critical condition in many articles.

\section{Preliminaries}

In this section, we state some known facts on concave operators; one can see [43-45].

Let $E$ be a Banach space, which has a partial order induced by a cone $K \subset E$, i.e., $x \leq y$ if and only if $y-x \in K$. For $x, y \in E$, the notation $x \sim y$ means that there are $\mu>0$ and $\nu>0$ such that $\mu x \leq y \leq \nu x$. Clearly, $\sim$ is an equivalence relation. For $h>\theta$ (i.e., $h \geq \theta$ and $h \neq \theta$ ), define $P_{h}=\{x \in E \mid x \sim h\}$. Clearly, $P_{h} \subset K$ is convex. Let $r \in K$ with $\theta \leq r \leq h$, and define $P_{h, r}=\left\{x \in E \mid x+r \in P_{h}\right\}$, that is,

$$
P_{h, r}=\{x \in E \mid \text { there exist } \mu=\mu(h, r, x)>0, \nu=\nu(h, r, x)>0 \text { such that } \mu h \leq x+r \leq \nu h\}
$$

Evidently, $P_{h}=P_{h, \theta}$.

Next, we list the definition of $\Psi-(h, r)$-concave operator and fixed point theorems that will play a key role in this paper.

Definition 1 (see [43]). Let $T: P_{h, r} \longrightarrow E$ be a given operator which satisfies, for $x \in P_{h, r}, \lambda \in(0,1)$, and there is $\Psi(\lambda)>\lambda$ such that $T(\lambda x+(\lambda-1) r) \geq \Psi(\lambda) T x+(\Psi(\lambda)-1) r$. Then, we call $T$ a $\Psi-(h, r)$-concave operator.

Lemma 1 (see [43]). Let $T$ be an increasing $\Psi-(h, r)$-concave operator and $P$ be normal, Th $\in P_{h, r}$. Then, $T$ has a unique fixed point $x^{*}$ in $P_{h, r}$. Take any $v_{0} \in P_{h, r}$, putting a sequence $v_{n}=T v_{n-1}, n=1,2, \ldots$; then, $\left\|v_{n}-x^{*}\right\| \longrightarrow 0$ as $n \longrightarrow \infty$.

Lemma 2 (see [44]). Let $T$ be an increasing $\Psi-(h, \theta)$-concave operator and $P$ be normal, Th $\in P_{h}$. Then, Thas a unique fixed point $x^{*}$ in $P_{h}$. Take any $v_{0} \in P_{h}$, making a sequence $v_{n}=T v_{n-1}, n=1,2, \ldots$; then, $\left\|v_{n}-x^{*}\right\| \longrightarrow 0$ as $n \longrightarrow \infty$.

\section{Main Results}

Let $E$ be an ordered Banach space, positive cone $K \subset E$ is normal, $\theta$ is the zero element of $E$, and $E$ has an identity element $e$, i.e., $e x=x, \forall x \in E$. In this section, let $J=[0,1]$, and we apply Lemmas 1 and 2 to study problem (4) in abstract Banach space $\mathscr{B}=C(J, E)$; the space of all continuous $E$-value functions on interval $J$ with the norm $\|u\|_{c}=\max [\|u(t)\|: t \in J]$. Then, $C(J, E)$ is an ordered Banach space induced by the cone $P=\{u \in C(J$ ,E) $\mid u(t) \in K, t \in J\}$, and $P$ is also a normal cone in $C(J, E)$.

Lemma 3 (see $[3,4]$ ). Assume $2<\beta \leq 3$ and $0<\rho<\beta$. Then, for every $y \in C(J, E)$, the problem of fractional linear differential equation in Banach spaces

$$
\begin{cases}-D_{0^{+}}^{\beta} u(t)=y(t), & 0<t<1 \\ u(0)=u^{\prime}(0)=\theta, & u(1)=\rho \int_{0}^{1} u(t) \mathrm{d} t,\end{cases}
$$

has a unique solution $u(t)=\int_{0}^{1} G(t, s) y(s) d s$, where

$$
G(t, s)= \begin{cases}\frac{t^{\beta-1}(1-s)^{\beta-1}(\beta-\rho+\rho s)-(\beta-\rho)(t-s)^{\beta-1}}{(\beta-\rho) \Gamma(\beta)}, & 0 \leq s \leq t \leq 1, \\ \frac{t^{\beta-1}(1-s)^{\beta-1}(\beta-\rho+\rho s)}{(\beta-\rho) \Gamma(\beta)}, & 0 \leq t \leq s \leq 1 .\end{cases}
$$


Lemma 4 (see $[3,4]$ ). Let $2<\beta \leq 3$ and $\rho \geq 0$. The function $G(t, s)$ given by (7) has the following properties:

(i) $G(t, s)>0$ for $t, s \in(0,1)$ and $\rho \in[0, \beta)$

(ii) $G(t, s) \leq(\beta /((\beta-\rho) \Gamma(\beta)))$ for $t, s \in(0,1)$ and $\rho \in[0, \beta)$

(iii) $\left(\left((1-s)^{\beta-1} \rho s\right) /((\beta-\rho) \Gamma(\beta))\right) t^{\beta-1} \leq G(t, s) \leq(((1-$ $\left.\left.s)^{\beta-1}(\beta-\rho+\rho s)\right) /((\beta-\rho) \Gamma(\beta))\right) t^{\beta-1}$, for $t, s \in$ $(0,1)$ and $\rho \in[0, \beta)$

Next, we intend to give some existence and uniqueness results for problem (4). We break our discussion up into two sections. Two cases of interest are given in Section 3.1 for $\gamma<0$ and in Section 3.2 for $\gamma \geq 0$, respectively. In Section 3.3, we discuss the existence and uniqueness corollaries for problem (4) in real space $C[J, \mathbf{R}]$.

3.1. Investigation for the Case $\gamma<0$. Let

$$
\int_{0}^{1} t(1-t)^{\beta-1} f(t, \theta) \mathrm{d} t \geq a_{1} \int_{0}^{1} g(t) \mathrm{d} t
$$

Theorem 1. Assume $e \in K$ and (H1)-(H3) are satisfied; then, problem (4) has a unique solution $u^{*}$ in $P_{h, r}$, where $r$ and $h$ are given in (8) and (9). Furthermore, set a sequence by

$$
\begin{array}{r}
v_{n}(t)=\int_{0}^{1} G(t, s) f\left(s, v_{n-1}(s)\right) \mathrm{d} s+\gamma \int_{0}^{1} G(t, s) g(s) \mathrm{d} s, \\
n=1,2, \ldots,
\end{array}
$$

$$
\begin{aligned}
r(t) & =-\gamma \int_{0}^{1} G(t, s) g(s) \mathrm{d} s, \quad t \in[0,1], \\
\hat{r} & =-\frac{\gamma \beta}{(\beta-\rho) \Gamma(\beta)} \int_{0}^{1} g(s) \mathrm{d} s, \\
h(t) & =H t^{\beta-1} \int_{0}^{1} g(s) \mathrm{d} s, \quad \text { with } H \geq-\frac{\gamma \beta}{(\beta-\rho) \Gamma(\beta)} .
\end{aligned}
$$

To prove the main results, we need the following assumptions:

(H1) $f:[0,1] \times[-\widehat{r}, \theta] \cup K \longrightarrow E$ is continuous and $g:[0,1] \longrightarrow K$ is continuous, and $f(t, u) \leq f(t, v)$ for $-\widehat{r} \leq u \leq v$.

(H2) For $\lambda \in(0,1)$ and $y \in[\theta, \widehat{r}]$, there is $1>\Psi(\lambda)>\lambda$ such that $f(t, \lambda x+(\lambda-1) y) \geq \Psi(\lambda) f(t, x), t \in$ $[0,1], x \in E$.

(H3) $f(t, \theta) \geq \theta$ with $f(t, \theta) \equiv \theta$ for $t \in[0,1]$, and there are two constants $a_{2} \geq a_{1}>0$ such that for any taken $v_{0} \in P_{h, r}$, and one has $v_{n}(t) \longrightarrow u^{*}(t)$ as $n \longrightarrow \infty$.

Proof. For $t \in[0,1]$, by Lemma 4 , one has

$$
\begin{aligned}
r(t) & =-\gamma \int_{0}^{1} G(t, s) g(s) \mathrm{d} s \leq-\gamma \int_{0}^{1} \frac{(1-s)^{\beta-1}(\beta-\rho+\rho s)}{(\beta-\rho) \Gamma(\beta)} t^{\beta-1} g(s) \mathrm{d} s, \\
& \leq-\frac{\gamma \beta}{(\beta-\rho) \Gamma(\beta)} \int_{0}^{1} g(s) \mathrm{d} s \cdot t^{\beta-1} \leq H t^{\beta-1} \int_{0}^{1} g(s) \mathrm{d} s=h(t) .
\end{aligned}
$$


Obviously, it follows $r(t) \geq \theta$, from $\gamma<0$ and Lemma 4 .

As $\theta \leq r(t) \leq h(t)$, we have $r \in P$. In the following, we will consider a set $P_{h, r}=\left\{u \in C(J, E) \mid u+r \in P_{h}\right\}$. By means of Lemma 3, if problem (4) has a solution $u$, then

$$
\begin{aligned}
u(t) & =\int_{0}^{1} G(t, s)[f(s, u(s))+\gamma g(s)] \mathrm{d} s \\
& =\int_{0}^{1} G(t, s) f(s, u(s)) \mathrm{d} s+\gamma \int_{0}^{1} G(t, s) g(s) \mathrm{d} s \\
& =\int_{0}^{1} G(t, s) f(s, u(s)) \mathrm{d} s-r(t) .
\end{aligned}
$$

So, for any $u \in P_{h_{h_{1}} r_{1}}$ and $t \in[0,1]$, we can define an operator by $T u(t)=\int_{0}^{1} G(t, s) f(s, u(s)) \mathrm{d} s-r(t)$. Hence, $u(t)$ is a solution of problem (4) if and only if $u$ is a fixed point of $T$.

Now, we show that $T: P_{h, r} \longrightarrow E$ satisfied the definition of $\Psi-(h, r)$-concave operator. For every $\lambda \in(0,1), u \in P_{h, r}$, and from condition (H2), one has

$$
\begin{aligned}
T(\lambda u+(\lambda-1) r)(t) & =\int_{0}^{1} G(t, s) f(s, \lambda u(s)+(\lambda-1) r(s)) \mathrm{d} s-r(t) \\
& \geq \Psi(\lambda) \int_{0}^{1} G(t, s) f(s, u(s)) \mathrm{d} s-\Psi(\lambda) r(t)+(\Psi(\lambda)-1) r(t) \\
& =\Psi(\lambda) T u(t)+(\Psi(\lambda)-1) r(t) .
\end{aligned}
$$

Therefore, we obtain

$$
\begin{array}{r}
T(\lambda u+(\lambda-1) r) \geq \Psi(\lambda) T u+(\Psi(\lambda)-1) r, \\
\lambda \in(0,1), u \in P_{h, r} .
\end{array}
$$

In view of Definition $1, T$ is a $\Psi-(h, r)$-concave operator.
Next, we prove that $T: P_{h, r} \longrightarrow E$ is increasing. Since $u \in P_{h, r}$, we get $u+r \in P_{h}$, so there is $\tau>0$ such that $u(t)+r(t) \geq \tau h(t)$; thus, we obtain

$$
u(t) \geq \tau h(t)-r(t) \geq-r(t) \geq-\widehat{r} .
$$

By condition (H1), T: $P_{h, r} \longrightarrow E$ is increasing.

The main step is to prove that $T h \in P_{h, r}$; in other words, we prove $T h+r \in P_{h}$. By (H1), (H3), and Lemma 4 , one has

$$
\begin{aligned}
T h(t)+r(t) & =\int_{0}^{1} G(t, s) f(s, h(s)) \mathrm{d} s-r(t)+r(t)=\int_{0}^{1} G(t, s) f(s, h(s)) \mathrm{d} s \\
& =\int_{0}^{1} G(t, s) f\left(s, H s^{\beta-1} \int_{0}^{1} g(z) \mathrm{d} z\right) \mathrm{d} s \\
& \leq \int_{0}^{1} \frac{(1-s)^{\beta-1}(\beta-\rho+\rho s)}{(\beta-\rho) \Gamma(\beta)} t^{\beta-1} f\left(s, H \int_{0}^{1} g(z) \mathrm{d} z\right) \mathrm{d} s \\
& \leq \frac{t^{\beta-1}}{(\beta-\rho) \Gamma(\beta)} \int_{0}^{1}(1-s)^{\beta-1}(\beta-\rho+\rho s) a_{2} g(s) \mathrm{d} s \leq \frac{a_{2} \beta}{(\beta-\rho) \Gamma(\beta)} t^{\beta-1} \int_{0}^{1} g(s) \mathrm{d} s \\
& =\frac{a_{2} \beta}{H(\beta-\rho) \Gamma(\beta)} H t^{\beta-1} \int_{0}^{1} g(s) \mathrm{d} s=\frac{a_{2} \beta}{H(\beta-\rho) \Gamma(\beta)} h(t), \\
T h(t)+r(t) & =\int_{0}^{1} G(t, s) f(s, h(s)) \mathrm{d} s=\int_{0}^{1} G(t, s) f\left(s, H s^{\beta-1} \int_{0}^{1} g(z) \mathrm{d} z\right) \mathrm{d} s \\
& \geq \int_{0}^{1} \frac{(1-s)^{\beta-1} \rho s}{(\beta-\rho) \Gamma(\beta)} t^{\beta-1} f(s, \theta) \mathrm{d} s=\frac{\rho}{(\beta-\rho) \Gamma(\beta)} \int_{0}^{1} s(1-s)^{\beta-1} f(s, \theta) \mathrm{d} s \cdot t^{\beta-1} \geq \frac{a_{1} \rho}{(\beta-\rho) \Gamma(\beta)} \int_{0}^{1} g(s) \mathrm{d} \cdot t^{\beta-1} \\
& =\frac{a_{1} \rho}{H(\beta-\rho) \Gamma(\beta)} H \int_{0}^{1} g(s) \mathrm{d} s \cdot t^{\beta-1}=\frac{a_{1} \rho}{H(\beta-\rho) \Gamma(\beta)} h(t) .
\end{aligned}
$$


Let

$$
\begin{aligned}
& l_{1}=\frac{a_{1} \rho}{H(\beta-\rho) \Gamma(\beta)}, \\
& l_{2}=\frac{a_{2} \beta}{H(\beta-\rho) \Gamma(\beta)} .
\end{aligned}
$$

Since $\Gamma(\beta)>0, H>0$, and from (H1) and (H3), we can easily get $l_{2} \geq l_{1}>0$. It follows that $T h+r \in P_{h}$.

Finally, by using Lemma 1, operator $T$ has a unique fixed point $u^{*}$ in $P_{h, r}$, and then,

$$
u^{*}(t)=\int_{0}^{1} G(t, s) f\left(s, u^{*}(s)\right) \mathrm{d} s-r(t), \quad t \in[0,1] .
$$

Consequently, $u^{*}(t)$ is the unique solution of problem (4) in $P_{h, r}$. Taking any $v_{0} \in P_{h, r}$, the sequence $v_{n}=T v_{n-1}$, $n=1,2, \ldots$, satisfies $v_{n} \longrightarrow u^{*}$ as $n \longrightarrow \infty$. That is,

$$
\begin{array}{r}
v_{n}(t)=\int_{0}^{1} G(t, s) f\left(s, v_{n-1}(s)\right) \mathrm{d} s+\gamma \int_{0}^{1} G(t, s) g(s) \mathrm{d} s, \\
n=1,2, \ldots,
\end{array}
$$

and $v_{n}(t) \longrightarrow u^{*}(t)$ as $n \longrightarrow \infty$.

3.2. Investigation for the Case $\gamma \geq 0$. In this section, we can get the unique result by using Lemma 2 . And, some other assumptions are listed in the following:

(H4) $f:[0,1] \times K \longrightarrow K$ is continuous with $f(t, \theta) \equiv \theta$ for $t \in[0,1], g:[0,1] \longrightarrow K$ is continuous.

(H5) For $t \in[0,1], f(t, x)$ is increasing with respect to the second variable, and there are $l_{1}, l_{2}>0$ such that

$$
\begin{aligned}
l_{1} e= & \frac{\rho}{(\beta-\rho) \Gamma(\beta)} \int_{0}^{1} s(1-s)^{\beta-1}[f(s, \theta)+\gamma g(s)] \mathrm{d} s, \\
l_{2} e= & \frac{1}{(\beta-\rho) \Gamma(\beta)} \int_{0}^{1}(1-s)^{\beta-1}(\beta-\rho+\rho s) \\
& \cdot[f(s, e)+\gamma g(s)] \mathrm{d} s .
\end{aligned}
$$

(H6) For $\lambda \in(0,1)$, there exists $1>\Psi(\lambda)>\lambda$ such that $f(t, \lambda x) \geq \Psi(\lambda) f(t, x), \forall t \in[0,1], x \in K$.

Theorem 2. Assume (H4)-(H6) are satisfied; then, for any taken $\gamma \geq 0$, problem (4) has a unique positive solution $u^{*}$ in $P_{h}$, where $h(t)=t^{\beta-1} e, t \in[0,1]$. Moreover, taking any initial value $v_{0} \in P_{h}$, the sequence

$$
v_{n}(t)=\int_{0}^{1} G(t, s)\left[f\left(s, v_{n-1}(s)\right)+\gamma g(s)\right] \mathrm{d} s, \quad n=1,2, \ldots,
$$

satisfies $v_{n}(t) \longrightarrow u^{*}(t)$ as $n \longrightarrow \infty$.

Proof. For $u \in P_{h}$, we define an operator $T$ by

$$
T u(t)=\int_{0}^{1} G(t, s)[f(s, u(s))+\gamma g(s)] \mathrm{d} s, \quad t \in[0,1],
$$

with $G(t, s)$ given as in Lemma 3.

From Lemma 3, $u(t)$ is the solution of problem (4) if and only if $u$ is the fixed point of $T$. Noting that $f(t, x) \geq \theta$ and $g(t) \geq \theta$, it is easy to check that $T: P \longrightarrow P$. From conditions $(\mathrm{H} 4)$ and $(\mathrm{H} 5)$, it is easy to prove that $T: P \longrightarrow P$ is increasing.

Next, we show that $T: P \longrightarrow P$ satisfied the definition of $\Psi-(h, \theta)$-concave operator. From (H6), for $\lambda \in(0,1)$ and $u \in P$, we obtain

$$
\begin{aligned}
T(\lambda u)(t) & =\int_{0}^{1} G(t, s)[f(s, \lambda u(s))+\gamma g(s)] \mathrm{d} s \\
& \geq \Psi(\lambda) \int_{0}^{1} G(t, s) f(s, u(s)) \mathrm{d} s+\gamma \int_{0}^{1} G(t, s) g(s) \mathrm{d} s \\
& \geq \Psi(\lambda) T u(t), \quad t \in[0,1] .
\end{aligned}
$$

Hence, $T(\lambda u) \geq \Psi(\lambda) T u, \forall u \in P$, and $\lambda \in(0,1)$.

In the following, we prove that $T h \in P_{h}$. Firstly, note that $h(t)=t^{\beta-1} e$, and from (H4), (H5), and Lemma 4, it follows that

$$
\begin{aligned}
T h(t) & =\int_{0}^{1} G(t, s)[f(s, h(s))+\gamma g(s)] \mathrm{d} s=\int_{0}^{1} G(t, s)\left[f\left(s, s^{\beta-1} e\right)+\gamma g(s)\right] \mathrm{d} s \\
& \geq \int_{0}^{1} \frac{(1-s)^{\beta-1} \rho s}{(\beta-\rho) \Gamma(\beta)} t^{\beta-1}[f(s, \theta)+\gamma g(s)] \mathrm{d} s \\
& =\frac{\rho}{(\beta-\rho) \Gamma(\beta)} \int_{0}^{1} s(1-s)^{\beta-1}[f(s, \theta)+\gamma g(s)] \mathrm{d} s \cdot t^{\beta-1} \\
& =l_{1} e \cdot t^{\beta-1}=l_{1} h(t) .
\end{aligned}
$$


Secondly, for $t \in[0,1]$, one has

Since $e \in P$, from (H5), it is easy to prove that

$$
\begin{aligned}
T h(t) & =\int_{0}^{1} G(t, s)\left[f\left(s, s^{\beta-1} e\right)+\gamma g(s)\right] \mathrm{d} s \\
& \leq \int_{0}^{1} \frac{(1-s)^{\beta-1}(\beta-\rho+\rho s)}{(\beta-\rho) \Gamma(\beta)} t^{\beta-1}[f(s, e)+\gamma g(s)] \mathrm{d} s \\
& =\frac{t^{\beta-1}}{(\beta-\rho) \Gamma(\beta)} \int_{0}^{1}(1-s)^{\beta-1}(\beta-\rho+\rho s)[f(s, e)+\gamma g(s)] \mathrm{d} s \\
& =l_{2} e \cdot t^{\beta-1}=l_{2} h(t) .
\end{aligned}
$$

$$
\begin{aligned}
\left(l_{2}-l_{1}\right) e= & l_{2} e-l_{1} e \\
= & \frac{1}{(\beta-\rho) \Gamma(\beta)} \int_{0}^{1}(1-s)^{\beta-1}(\beta-\rho+\rho s)[f(s, e)+\gamma g(s)] \mathrm{d} s-\frac{\rho}{(\beta-\rho) \Gamma(\beta)} \int_{0}^{1} s(1-s)^{\beta-1}[f(s, \theta)+\gamma g(s)] \mathrm{d} s \\
= & \frac{1}{\Gamma(\beta)} \int_{0}^{1}(1-s)^{\beta-1}[f(s, e)+\gamma g(s)] \mathrm{d} s+\frac{\rho}{(\beta-\rho) \Gamma(\beta)} \int_{0}^{1} s(1-s)^{\beta-1}[f(s, e)+\gamma g(s)] \mathrm{d} s \\
& -\frac{\rho}{(\beta-\rho) \Gamma(\beta)} \int_{0}^{1} s(1-s)^{\beta-1}[f(s, \theta)+\gamma g(s)] \mathrm{d} s \\
= & \frac{1}{\Gamma(\beta)} \int_{0}^{1}(1-s)^{\beta-1}[f(s, e)+\gamma g(s)] \mathrm{d} s+\frac{\rho}{(\beta-\rho) \Gamma(\beta)} \int_{0}^{1} s(1-s)^{\beta-1}[f(s, e)-f(s, \theta)] \mathrm{d} s \geq \theta
\end{aligned}
$$

Hence $l_{2} \geq l_{1}>0$. It follows that $T h \in P_{h}$. Finally, by using Lemma 2 , the operator $T$ has a unique fixed point $u^{*}$ in $P_{h}$, and

$$
u^{*}(t)=\int_{0}^{1} G(t, s)\left[f\left(s, u^{*}(s)\right) \mathrm{d} s+\gamma g(s)\right] \mathrm{d} s, \quad t \in[0,1] .
$$

Consequently, $u^{*}(t)$ is the unique solution of problem (4) in $P_{h}$. For $v_{0} \in P_{h}$, the sequence $v_{n}=T v_{n-1}, n=1,2, \ldots$, satisfies $v_{n} \longrightarrow u^{*}$ as $n \longrightarrow \infty$. That is,

$$
v_{n}(t)=\int_{0}^{1} G(t, s)\left[f\left(s, v_{n-1}(s)\right)+\gamma g(s)\right] \mathrm{d} s, \quad n=1,2, \ldots,
$$

satisfies $v_{n}(t) \longrightarrow u^{*}(t)$ as $n \longrightarrow \infty$.

Remark 1. The form of problem (4) is more general. Our method is new to study fractional differential equations in Banach spaces, which guarantees the existence and uniqueness of solutions or positive solutions. We also can make an iteration to approximate the unique solution.
3.3. Direct Corollaries. Let $E=\mathbf{R}, \mathscr{B}=C[0,1]$, and $P=\{u \in C[0,1], u(t) \geq 0, t \in[0,1]\}$. We can get some obvious results from Theorems 1 and 2 . Set

$$
\begin{aligned}
r(t) & =-\gamma \int_{0}^{1} G(t, s) g(s) \mathrm{d} s, \quad \forall t \in[0,1], \\
\widehat{r} & =-\frac{\gamma \beta}{(\beta-\rho) \Gamma(\beta)} \int_{0}^{1} g(s) \mathrm{d} s, \\
h(t) & =H t^{\beta-1} \int_{0}^{1} g(s) \mathrm{d} s, \text { with } H \geq-\frac{\gamma \beta}{(\beta-\rho) \Gamma(\beta)} .
\end{aligned}
$$

(H1') $f:[0,1] \times[-\widehat{r},+\infty) \longrightarrow(-\infty,+\infty)$ is continuous, $f(t, u) \leq f(t, v)$ for $-\widehat{r} \leq u \leq v<+\infty$, and $g:[0,1] \longrightarrow[0,+\infty)$ is continuous.

(H2') For $\lambda \in(0,1)$ and $y \in[0, \hat{r}]$, there is $\Psi(\lambda)>\lambda$ such that $f(t, \lambda x+(\lambda-1) y) \geq \Psi(\lambda) f(t, x), \forall t \in[0,1]$ ,$x \in(-\infty,+\infty)$.

(H3') $f(t, 0) \geq 0, f(t, 0) \equiv 0$ for $t \in[0,1]$, and there are two constants $a_{2} \geq a_{1}>0$ such that 


$$
\int_{0}^{1} t(1-t)^{\beta-1} f(t, 0) \mathrm{d} t \geq a_{1} \int_{0}^{1} g(t) \mathrm{d} t, f\left(t, H \int_{0}^{1} g(s) \mathrm{d} s\right) \leq a_{2} g(t), \quad \forall t \in[0,1] .
$$

Corollary 1. Let $\beta \in(2,3]$ and $\rho \in(0, \beta)$. Assume that (H1')-(H3') hold; then, for $\gamma<0$, the boundary value problem

$$
\begin{cases}-D_{0^{+}}^{\beta} u(t)=f(t, u(t))+\gamma g(t), & 0 \leq t \leq 1, \\ u(0)=u^{\prime}(0)=0, & u(1)=\rho \int_{0}^{1} u(t) \mathrm{d} t,\end{cases}
$$

has a unique solution $u^{*}$ in $P_{h, r}$, where $r$ and $h$ are given in (30 and 31). Furthermore, set a sequence

$$
v_{n}(t)=\int_{0}^{1} G(t, s) f\left(s, v_{n-1}(s)\right) \mathrm{d} s+\gamma \int_{0}^{1} G(t, s) g(s) \mathrm{d} s, \quad n=1,2, \ldots,
$$

for any taken $v_{0} \in P_{h, r}$, and one has $v_{n}(t) \longrightarrow u^{*}(t)$ as $n \longrightarrow \infty$.

Corollary 2. Let $\beta \in(2,3]$ and $\rho \in(0, \beta)$. Assume that

$\left(H 4^{\prime}\right) f:[0,1] \times[0,+\infty) \longrightarrow[0,+\infty)$ is continuous with $f(t, 0) \equiv 0$ for $t \in[0,1]$ and $g:[0,1] \longrightarrow$ $[0,+\infty)$ is continuous

$\left(H 5^{\prime}\right)$ For $t \in[0,1], f(t, x)$ is increasing in the second variable

(H6') For $\lambda \in(0,1)$, there exists $\Psi(\lambda)>\lambda$ such that $f(t, \lambda x) \geq \Psi(\lambda) f(t, x), \forall t \in[0,1], x \in[0,+\infty) ;$ then, for $\gamma \geq 0$, the boundary value problem

$$
\begin{cases}-D_{0^{+}}^{\beta} u(t)=f(t, u(t))+\gamma g(t), & 0<t<1, \\ u(0)=u^{\prime}(0)=0, & u(1)=\rho \int_{0}^{1} u(t) \mathrm{d} t,\end{cases}
$$

has a unique solution $u^{*}$ in $P_{h}$, where $h(t)=t^{\beta-1}$. Furthermore, put a sequence by

$$
v_{n}(t)=\int_{0}^{1} G(t, s)\left[f\left(s, v_{n-1}(s)\right)+\gamma g(s)\right] \mathrm{d} s, \quad n=1,2, \ldots,
$$

for any taken $v_{0} \in P_{h}$, and one has $v_{n}(t) \longrightarrow u^{*}(t)$ as $n \longrightarrow \infty$.

\section{Examples}

To illustrate our main results, we present two examples.

Example 1. Consider the following boundary value problem:

$$
\begin{cases}-D_{0^{+}}^{(5 / 2)} u(t)=f(t, u(t))-2 t, & 0<t<1, \\ u(0)=u^{\prime}(0)=0, & u(1)=\frac{1}{2} \int_{0}^{1} u(t) \mathrm{d} t,\end{cases}
$$

where

$$
f(t, u)=\left\{\left(\frac{8}{35} u+\frac{15}{7 \Gamma(7 / 2)}\right) t^{(5 / 2)}-\left(\frac{16}{25} u+\frac{2}{\Gamma(7 / 2)}\right) t^{(7 / 2)}\right\}^{(1 / 3)},
$$

and $\quad \beta=(5 / 2), \rho=(1 / 2), \gamma=-2, g(t)=t$, and $t \in[0,1]$. After a simple calculation,

$$
r(t)=-\gamma \int_{0}^{1} G(t, s) g(s) \mathrm{d} s=\frac{2 t^{(5 / 2)}(1-t)}{\Gamma(7 / 2)}+\frac{t^{(5 / 2)}}{2 \Gamma(9 / 2)}=\left[\frac{2}{\Gamma(7 / 2)}+\frac{1}{7 \Gamma(7 / 2)}\right] t^{(5 / 2)}-\frac{2}{\Gamma(7 / 2)} t^{(7 / 2)}, \quad t \in[0,1]
$$

where $G(t, s)$ is given as Lemma 1. Moreover, $h(t)=H t^{(3 / 2)}$ with $H \geq(5 /(4 \Gamma(5 / 2))), \forall t \in[0,1]$. Then, 


$$
\begin{aligned}
& r(t)=\frac{2 t^{(5 / 2)}(1-t)}{\Gamma(7 / 2)}+\frac{t^{(5 / 2)}}{2 \Gamma(9 / 2)} \geq 0, \\
& r(t)=\left[\frac{2}{\Gamma(7 / 2)}+\frac{1}{7 \Gamma(7 / 2)}\right] t^{(5 / 2)}-\frac{2}{\Gamma(7 / 2)} t^{(7 / 2)} \leq\left(2+\frac{1}{7}\right) \frac{1}{\Gamma(7 / 2)} t^{(5 / 2)}<\frac{5}{4 \Gamma(5 / 2)} t^{(3 / 2)} \leq H t^{(3 / 2)}=h(t) .
\end{aligned}
$$

Furthermore, $\hat{r}=(5 /(4 \Gamma(5 / 2)))$. We can see that $f:[0,1] \times[-(5 /(4 \Gamma(5 / 2))),+\infty) \longrightarrow(-\infty,+\infty)$ is continuous and increasing in the second variable, and

$$
\begin{aligned}
& f(t, 0)=\left[\frac{15}{7 \Gamma(7 / 2)} t\right. \\
& \text { Thus, conditions ( } \mathrm{H} 1 \\
& \text { can be expressed as } \\
& \text { d } \\
& -\frac{16}{25} t^{(7 / 2)}, t \in[0,1] .
\end{aligned}
$$

From the Remark 4 in [43], we obtain

with $f(t, 0) \equiv 0, t \in[0,1]$. Thus, conditions (H1') and (H3') are satisfied. Obviously, it can be expressed as $f(t, u(t))=$ $[(r(t) / \widehat{r}) u(t)+r(t)]^{(1 / 3)}$ and

$$
\frac{r(t)}{\widehat{r}}=\frac{8}{35} t^{(5 / 2)}-\frac{16}{25} t^{(7 / 2)}, t \in[0,1] .
$$

where $\Psi(\lambda)=\lambda^{(1 / 3)}>\lambda, \lambda \in(0,1)$; then, condition (H1') is satisfied. In view of Corollary 1 , problem (37) has a unique solution $u^{*}$ in $P_{h, r}$. For $v_{0} \in P_{h, r}$, let

$$
\begin{aligned}
v_{n}(t)= & \int_{0}^{1} G(t, s) f\left(s, v_{n-1}(s)\right) \mathrm{d} s+(-2) \int_{0}^{1} G(t, s) t \mathrm{~d} s \\
= & \int_{0}^{1} G(t, s)\left\{\left(\frac{8}{35} v_{n-1}(s)+\frac{15}{7 \Gamma(7 / 2)}\right) s^{(5 / 2)}-\left(\frac{16}{25} v_{n-1}(s)+\frac{2}{\Gamma(7 / 2)}\right) s^{(7 / 2)}\right\} \mathrm{d} s \\
& -\left[\frac{2}{\Gamma(7 / 2)}+\frac{1}{7 \Gamma(7 / 2)}\right] t^{(5 / 2)}+\frac{2}{\Gamma(7 / 2)} t^{(7 / 2)}, \quad n=1,2, \ldots
\end{aligned}
$$

We have $v_{n}(t) \longrightarrow u^{*}(t)$ as $n \longrightarrow \infty, t \in[0,1]$.

Example 2. Consider the following boundary value problem:

$$
\begin{cases}-D_{0^{+}}^{(5 / 2)} u(t)=\left\{\left(\frac{5}{3} t^{2}+t\right)(u+2)\right\}^{(5 / 2)}+\gamma g(t), & 0<t<1 \\ u(0)=u^{\prime}(0)=0, & u(1)=\frac{1}{2} \int_{0}^{1} u(t) \mathrm{d} t\end{cases}
$$

where $f(t, u)=\left\{\left((5 / 3) t^{2}+t\right)(u+2)\right\}^{(2 / 5)}, t \in[0,1]$, and $\beta=(5 / 2), \rho=(1 / 2), \gamma=0$, and $g(t) \geq 0, t \in[0,1]$ are continuous. Take $h(t)=t^{\beta-1}, t \in[0,1]$. It is clear that $f:[0,1] \times[0,+\infty) \longrightarrow[0,+\infty)$ is continuous and increasing in the second variable, $f(t, 0)=\left((10 / 3) t^{2}+2 t\right)^{(2 / 5)} \geq 0$ with $f(t, 0) \equiv 0, t \in[0,1]$, and thus, conditions ( $\left.\mathrm{H} 4^{\prime}\right)$ and (H5') are satisfied. Next, for $\lambda \in(0,1)$, 


$$
\begin{aligned}
f(t, \lambda u) & =\left\{\left(\frac{5}{3} t^{2}+t\right)(\lambda u+2)\right\}^{(2 / 5)} \geq\left\{\left(\frac{5}{3} t^{2}+t\right)(\lambda u+2 \lambda)\right\}^{(2 / 5)}, \\
& =\lambda^{(2 / 5)}\left\{\left(\frac{5}{3} t^{2}+t\right)(u+2)\right\}^{(2 / 5)}, \quad t \in[0,1],
\end{aligned}
$$

where $\Psi(\lambda)=\lambda^{(2 / 5)}>\lambda, \lambda \in(0,1)$; then, condition (H6') is satisfied. In view of Corollary 2 , problem (45) has a unique solution $u^{*}$ in $P_{h}$. For $v_{0} \in P_{h}$, set

$$
v_{n}(t)=\int_{0}^{1} G(t, s)\left\{\left(\frac{5}{3} t^{2}+t\right)\left(v_{n-1}(t)+2\right)\right\}^{(2 / 5)}, \quad n=1,2, \ldots,
$$

And we have $v_{n}(t) \longrightarrow u^{*}(t)$ as $n \longrightarrow \infty, t \in[0,1]$.

\section{Conclusion}

In this paper, we study an integral boundary value problem (4) with sign-changed parameter in Banach spaces. By taking two functions $r$ and $h$ and using fixed point theorems of increasing $\Psi-(h, r)$-concave operators defined on ordered set $P_{h, r}$, we establish some new existence and uniqueness criteria for problem (4) dependent on different parameters, which also give some different answers to the same type of fractional differential equations' boundary value problem in the literature [3]. Our results can guarantee the existence of a unique solution with not supposing the existence of upperlower solutions. As applications, two good examples are presented to illustrate the main results.

\section{Data Availability}

No data were used to support the findings of the study.

\section{Conflicts of Interest}

The authors declare that they have no conflicts of interest.

\section{Authors' Contributions}

The authors declare that the study was realized in collaboration with the same responsibility. All authors read and approved the final manuscript.

\section{Acknowledgments}

This work was supported by Shanxi Province Science Foundation (201901D111020) and Graduate Science and Technology Innovation Project of Shanxi (2019BY014).

\section{References}

[1] B. Ahmad and S. Sivasundaram, "On four-point nonlocal boundary value problems of nonlinear integro-differential equations of fractional order," Applied Mathematics and Computation, vol. 217, no. 2, pp. 480-487, 2010.

[2] Z. Bai, "On positive solutions of a nonlocal fractional boundary value problem," Nonlinear Analysis: Theory, Methods \& Applications, vol. 72, no. 2, pp. 916-924, 2010.
[3] P. Chen and Y. Gao, "Positive solutions for a class of nonlinear fractional differential equations with nonlocal boundary value conditions," Positivity, vol. 22, no. 3, pp. 761-772, 2018.

[4] A. Cabada and Z. Hamdi, "Nonlinear fractional differential equations with integral boundary value conditions," Applied Mathematics and Computation, vol. 228, pp. 251-257, 2014.

[5] Y. Wang, L. Liu, and Y. Wu, "Positive solutions for a nonlocal fractional differential equation," Nonlinear Analysis: Theory, Methods \& Applications, vol. 74, no. 11, pp. 3599-3605, 2011.

[6] C. Zhai, W. Wang, and H. Li, "A uniqueness method to a new Hadamard fractional differential system with four-point boundary conditions," Journal of Inequalities and Applications, vol. 2018, p. 1, 2018.

[7] C. Zhai and W. Wang, "Properties of positive solutions for m-point fractional differential equations on an infinite interval," Revista de la Real Academia de Ciencias Exactas, Físicas y Naturales. Serie A. Matemáticas, vol. 113, no. 2, pp. 1289-1298, 2019.

[8] C. Zhai and J. Ren, "The unique solution for a fractionalqdifference equation with three-point boundary conditionsdifference equation with three-point boundary conditions," Indagationes Mathematicae, vol. 29, no. 3, pp. 948-961, 2018.

[9] Z.-W. Lv, J. Liang, and T.-J. Xiao, "Solutions to the Cauchy problem for differential equations in Banach spaces with fractional order," Computers \& Mathematics with Applications, vol. 62, no. 3, pp. 1303-1311, 2011.

[10] B. Ahmad, A. Alsaedi, A. Alsaedi, and S. K. Ntouyas, "Fractional order nonlinear mixed coupled systems with coupled integro-differential boundary conditions," Journal of Applied Analysis \& Computation, vol. 10, no. 3, pp. 892-903, 2020.

[11] S. Meng and Y. Cui, "Multiplicity results to a conformable fractional differential equations involving integral boundary condition," Complexity, vol. 2019, Article ID 8402347, 2019.

[12] W. Wang, "Properties of Green's function and the existence of different types of solutions for nonlinear fractional BVP with a parameter in integral boundary conditions, Bound," Boundary Value Problems, vol. 2019, p. 76, 2019.

[13] Y. Sun and M. Zhao, "Positive solutions for a class of fractional differential equations with integral boundary conditions," Applied Mathematics Letters, vol. 34, pp. 17-21, 2014.

[14] S. K. Ntouyas and H. H. Al-Sulami, "A study of coupled systems of mixed order fractional differential equations and inclusions with coupled integral fractional boundary conditions," Advances in Difference Equations, vol. 2020, p. 73, 2020.

[15] J. Tan, M. Li, and A. Pan, "Uniqueness of iterative positive solution to nonlinear fractional differential equations with negatively perturbed term," Journal of Function Spaces, vol. 2020, p. 6, Article ID 5747425, 2020.

[16] Y. Li, H. Sun, and Q. Zhang, "Existence of solutions to fractional boundary-value problems with a parameter," Electronic Journal of Differential Equations, vol. 141, pp. 1783-1812, 2013.

[17] K. Zhao, "Triple positive solutions for two classes of delayed nonlinear fractional FDEs with nonlinear integral boundary 
value conditions," Boundary Value Problems, vol. 2015, p. 181, 2015.

[18] C. Zhai and J. Ren, "Positive and negative solutions of a boundary value problem for a fractional-difference equation," Advances in Difference Equations, vol. 2017, p. 82, 2017.

[19] X. Li, X. Liu, M. Jia, Y. Li, and S. Zhang, "Existence of positive solutions for integral boundary value problems of fractional differential equations on infinite interval," Mathematical Methods in the Applied Sciences, vol. 40, no. 6, pp. 1892-1904, 2017.

[20] B. Ahmad and J. J. Nieto, "Existence results for nonlinear boundary value problems of fractional integrodifferential equations with integral boundary conditions," Boundary Value Problems, vol. 2009, pp. 1-11, Article ID 708576, 2009.

[21] J. Wang, M. Fekan, and Y. Zhou, "A survey on impulsive fractional differential equations," Fractional Calculus and Applied Analysis, vol. 19, pp. 806-831, 2016.

[22] W. Wang and X. Guo, "Eigenvalue problem for fractional differential equations with nonlinear integral and disturbance parameter in boundary conditions, Bound," Boundary Value Problems, vol. 17, no. 6, pp. 2530-2538, 2016.

[23] Y. Wang and L. Liu, "Uniqueness and existence of positive solutions for the fractional integro-differential equation, Bound," Boundary Value Problems, vol. 2017, no. 12, 17 pages, 2017.

[24] J. Wang, S. Peng, and D. O’Regan, “Local stable manifold of Langevin differential equations with two fractional derivatives," Advances in Difference Equations, vol. 2017, p. 355, 2017.

[25] Y. Ding, J. Jiang, D. O’Regan, and J. Xu, "Positive solutions for a system of Hadamard-type fractional differential equations with semipositone nonlinearities," Complexity, p. 14, Article ID 9742418, 2020.

[26] H. Wang and L. Zhang, "The solution for a class of sum operator equation and its application to fractional differential equation boundary value problems, Bound," Boundary Value Problems, vol. 2015, p. 203, 2015.

[27] X. Hao, "Positive solution for singular fractional differential equations involving derivatives," Advances in Difference Equations, vol. 2016, p. 139, 2016.

[28] N. Xu and W. Liu, "Iterative solutions for a coupled system of fractional differential-integral equations with two-point boundary conditions," Applied Mathematics and Computation, vol. 244, pp. 903-911, 2014.

[29] C. Yang, C. Zhai, and L. Zhang, "Local uniqueness of positive solutions for a coupled system of fractional differential equations with integral boundary conditions," Advances in Difference Equations, vol. 2017, p. 282, 2017.

[30] C. Yang, "Existence and uniqueness of positive solutions for boundary value problems of a fractional differential equation with a parameter," Hacettepe Journal of Mathematics and Statistics, vol. 44, no. 3, pp. 665-673, 2015.

[31] B. Zhu, L. Liu, and Y. Wu, "Local and global existence of mild solutions for a class of nonlinear fractional reaction-diffusion equations with delay," Applied Mathematics Letters, vol. 61, pp. 73-79, 2016.

[32] L. Zhang, B. Ahmad, G. Wang, and R. P. Agarwal, "Nonlinear fractional integro-differential equations on unbounded domains in a Banach space," Journal of Computational and Applied Mathematics, vol. 249, pp. 51-56, 2013.

[33] X. Zhang, L. Liu, Y. Wu, and B. Wiwatanapataphee, "Nontrivial solutions for a fractional advection dispersion equation in anomalous diffusion," Applied Mathematics Letters, vol. 66, pp. 1-8, 2017.
[34] X. Zhang, C. Mao, L. Liu, and Y. Wu, "Exact iterative solution for an abstract fractional dynamic system model for bioprocess," Qualitative Theory of Dynamical Systems, vol. 16, no. 1, pp. 205-222, 2017.

[35] X. Zhang, L. Liu, Y. Wu, and B. Wiwatanapataphee, "The spectral analysis for a singular fractional differential equation with a signed measure," Applied Mathematics and Computation, vol. 257, pp. 252-263, 2015.

[36] H. Zhang, Y. Li, and J. Xu, "Positive solutions for a system of fractional integral boundary value problems involving Hadamard-type fractional derivatives," Complexity, p. 11, Article ID 2671539, 2019.

[37] G. Wang, X. Ren, and D. Baleanu, "Maximum principle for Hadamard fractional differential equations involving fractional Laplace operator," Mathematical Methods in the Applied Sciences, vol. 43, no. 5, pp. 2646-2655, 2020.

[38] J. Xu, J. Jiang, and D. O’Regan, "Positive Solutions for a class of p-Laplacian Hadamard fractional-order three-point boundary value problems," Mathematics, vol. 8, no. 3, 2020.

[39] X. Liu and M. Jia, "The method of lower and upper solutions for the general boundary value problems of fractional differential equations with p-Laplacian," Advances in Difference Equations, vol. 28, 2018.

[40] L. Shu, X.-B. Shu, and J. Mao, "Approximate controllability and existence of mild solutions for Riemann-Liouville fractional stochastic evolution equations with nonlocal conditions of order 1," Fractional Calculus and Applied Analysis, vol. 22, no. 4, pp. 1086-1112, 2019.

[41] Y. Guo, X. Shu, Y. Li, and F. Xu, "The existence and HyersUlam stability of solution for an impulsive Riemann-Liouville fractional neutral functional stochastic differential equation with infinite delay of order," Boundary Value Problems, vol. 59, 2019.

[42] X.-B. Shu and Y. Shi, "A study on the mild solution of impulsive fractional evolution equations," Applied Mathematics and Computation, vol. 273, pp. 465-476, 2016.

[43] C. Zhai and L. Wang, " $\varphi-(\mathrm{h}, \mathrm{e})$-concave operators and applications," Journal of Mathematical Analysis and Applications, vol. 454, no. 2, pp. 571-584, 2017.

[44] C. Zhai and F. Wang, "Properties of positive solutions for the operator equation and applications to fractional differential equations with integral boundary conditions," Advances in Difference Equations, vol. 2015, p. 366, 2015.

[45] C. Zhai and J. Ren, "Some properties of sets, fixed point theorems in ordered product spaces and applications to a nonlinear system of fractional differential equations," Topological Methods in Nonlinear Analysis, vol. 49, no. 2, pp. 625-645, 2017. 